\title{
Anti-HER2 therapy-associated cardiotoxicity in breast cancer patients: analysis of real-world data from a UK cancer centre
}

\author{
Authors: Agathoklis Efthymiadis ${ }^{\mathrm{A}}$ and May Teoh ${ }^{\mathrm{B}}$
}

\section{Introduction}

Cardiac adverse events are a recognised toxicity for patients with breast cancer receiving anti-human epidermal growth factor receptor-2 (HER2) therapy. ${ }^{1}$ Large phase III clinical trials have shown an incidence of left ventricular ejection fraction (LVEF) decrease $>10 \%$ of $7.1-18.6 \%$ and overt heart failure of $1.7-4.1 \% .^{2,3}$ Retrospective analyses from large registries such as the Surveillance, Epidemiology and End Results (SEER) and Cancer Research Network have reported an even higher incidence of heart failure at $41.9 \%$ and $20.1 \%$, respectively. ${ }^{4,5}$ We conducted a retrospective analysis to evaluate the incidence of cardiac adverse events for patients receiving anti-HER2 therapy at St Luke's Cancer Centre (SLCC) and Ashford and St Peter's Hospital (ASPH). The aims of the study were to provide real-world data on cardiotoxicity with anti-HER2 therapy and to evaluate adherence to the cardiac surveillance protocol.

\section{Materials and methods}

Patients with breast cancer receiving anti-HER2 therapy between June 2018 and June 2019 at SLCC and ASPH were included in the analysis. Data on patient demographics, chemotherapy and anti-HER2 therapy details, and LVEF values were obtained from the systemic anti-cancer therapy electronic prescribing system and patient records.

\section{Results and discussion}

One hundred and twenty-three female patients were analysed; the median age was 56 years (range 29-82 years); 25 (23.8\%) patients had at least one cardiac comorbidity, with hypertension being the most common; $66 \%$ of patients were treated with curative intent; $40 \%$ of patients received combined anthracycline-taxane chemotherapy, while $60 \%$ received only taxane-based chemotherapy; $55.3 \%$ received dual anti-HER therapy with pertuzumab and trastuzumab, while $44.7 \%$ were treated with trastuzumab alone. The median number of antiHER2 therapy doses was 16 (range 3-93). An asymptomatic decrease in LVEF was recorded in 33 patients (26.8\%). Of these, 19 patients had a decrease in LVEF $>10$ points below baseline, six had an LVEF decrease to $<50 \%$ and eight had

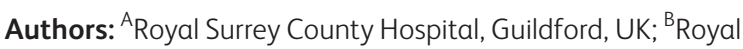
Surrey NHS Foundation Trust, UK both a decrease in LVEF $>10$ points and $<50 \%$. The median time from start of treatment to LVEF decrease was 5 months (range 2-36 months). At the time of reporting, the LVEF of 22 patients $(66.7 \%)$ had recovered to baseline. There were no cases of symptomatic heart failure. Three patients had delays in their treatment due to the LVEF drop and two stopped treatment early. LVEF decrease was more common in patients receiving palliative treatment ( $35.7 \%$ vs $22.5 \%$ ) and anthracycline chemotherapy ( $28.3 \%$ vs $24.6 \%$ ). No association was found between LVEF decrease and cardiac comorbidity or type of antiHER2 therapy. Adherence to the cardiac surveillance protocol was $76.7 \%$. The most common deviation from protocol was a delay in the cardiac function assessment at 4 months.

\section{Conclusions}

In this real-world analysis of cardiotoxicity with anti-HER2 therapy, the incidence of significant cardiac adverse events and interruption or discontinuation of therapy was very low, confirming the safety of this treatment modality. Asymptomatic decrease in LVEF was associated with palliative treatment intent and anthracycline-based chemotherapy. Adherence to the cardiac surveillance protocol is important, as the majority of LVEF decreases occur within the first 5 months of therapy and are potentially reversible with appropriate interventions.

\section{Conflicts of interest}

None declared.

\section{References}

1 Bloom M, Hamo C, Cardinale D et al. Cancer therapy-related cardiac dysfunction and heart failure: Part 1: definitions, pathophysiology, risk factors, and imaging. Circ Heart Fail 2016;9:e002661.

2 Procter M, Suter T, Ede Azambuja et al. Longer-term assessment of trastuzumab-related cardiac adverse events in the Herceptin Adjuvant (HERA) trial. J Clin Oncol 2010;28:3422-8.

3 Advani P, Ballman K, Dockter T et al. Long-term cardiac safety analysis of NCCTG N9831 (Alliance) adjuvant trastuzumab trial. $J$ Clin Oncol 2016;34:581-7.

4 Bowles E, Wellman R et al. Risk of heart failure in breast cancer patients after anthracycline and trastuzumab treatment: A retrospective cohort study. J Natl Cancer Inst 2012;104:1293-305.

5 Chen J, Long J, Hurria A et al. Incidence of heart failure or cardiomyopathy after adjuvant trastuzumab therapy for breast cancer. $J$ Am Coll Cardiol 2012;60:2504-12. 